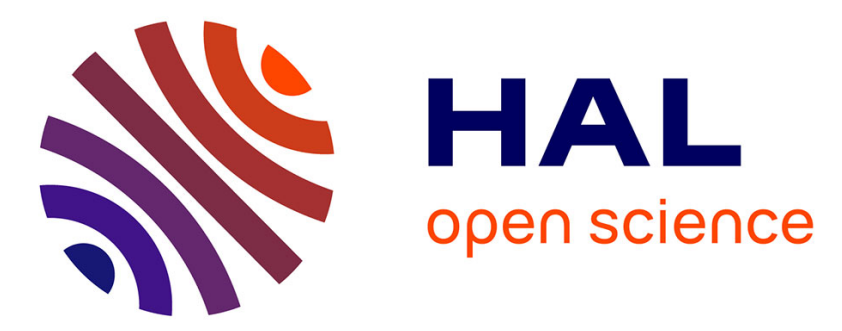

\title{
Passive Acoustic Leak Detection for Sodium Cooled Fast Reactors Using Hidden Markov Models
}

\author{
A. Riber Marklund, S. Kishore, V. Prakash, K. K. Rajan, F. Michel
}

\section{To cite this version:}

A. Riber Marklund, S. Kishore, V. Prakash, K. K. Rajan, F. Michel. Passive Acoustic Leak Detection for Sodium Cooled Fast Reactors Using Hidden Markov Models. ANIMMA 2015 - 4th International Conference on Advancements in Nuclear Instrumentation Measurement Methods and their Applications, Apr 2015, Lisbon, Portugal. 10.1109/ANIMMA.2015.7465560 . cea-02509266

\section{HAL Id: cea-02509266 https://hal-cea.archives-ouvertes.fr/cea-02509266}

Submitted on 16 Mar 2020

HAL is a multi-disciplinary open access archive for the deposit and dissemination of scientific research documents, whether they are published or not. The documents may come from teaching and research institutions in France or abroad, or from public or private research centers.
L'archive ouverte pluridisciplinaire HAL, est destinée au dépôt et à la diffusion de documents scientifiques de niveau recherche, publiés ou non, émanant des établissements d'enseignement et de recherche français ou étrangers, des laboratoires publics ou privés. 


\title{
Passive Acoustic Leak Detection for Sodium Cooled Fast Reactors Using Hidden Markov Models
}

\author{
A. Riber Marklund, S. Kishore, V. Prakash, K. K. Rajan, F. Michel
}

\begin{abstract}
Acoustic leak detection for steam generators of sodium fast reactors have been an active research topic since the early 1970s and several methods have been tested over the years. Inspired by its success in the field of automatic speech recognition, we here apply hidden Markov models (HMM) in combination with Gaussian mixture models (GMM) to the problem. To achieve this, we propose a new feature calculation scheme, based on the temporal evolution of the power spectral density (PSD) of the signal. Using acoustic signals recorded during steam/water injection experiments done at the Indira Gandhi Centre for Atomic Research (IGCAR), the proposed method is tested. We perform parametric studies on the HMM+GMM model size and demonstrate that the proposed method a) performs well without a priori knowledge of injection noise, b) can incorporate several noise models and c) has an output distribution that simplifies false alarm rate control.
\end{abstract}

\section{INTRODUCTION}

SODIUM fast reactors (SFRs) represent a possible option for $S_{\text {reaching the standards for future nuclear power concepts }}$ proposed by the Generation IV forum [1]. Inherent in the SFR design are however risks related to the interaction between sodium and water in the turbine circuit. Ongoing research and development performed at the CEA therefore aims to develop a sodium-nitrogen energy conversion system for SFRs. In any case, a leak between the secondary and tertiary sides, i.e. inside steam generators or sodium-nitrogen heat exchangers will remain an accident precursor which has to be detected in a fast and reliable way.

Research on acoustic leak detection (ALD) for this purpose has been ongoing for many years. Both active and passive methods may be needed in the final implementation

Manuscript received March 31, 2015. This study was performed within the framework of a collaboration project between KTH and CEA on the instrumentation and safety of sodium cooled reactors. The work was supported by the Swedish Research Council, grant number B0774801.

A. Riber Marklund is with the Royal Institute of Technology (KTH), currently on secondment to the Commisariat a l'Energie Atomique et aux Energies Alternatives (CEA), Cadarache center DEN/DTN/STCP/LIET Bâtiment 202, 13108 St Paul-lez-Durance, France (telephone: +33 (0)4 42 255726 , e-mail: andersrg@kth.se).

S. Kishore is with the Fast Reactor Technology Group of IGCAR (e-mail: skishore@igcar.gov.in).

V. Prakash is with the Vibrations Diagnostics Division, Fast Reactor Technology Group of IGCAR (telephone: +91 44274800 86, e-mail: prakash@igcar.gov.in).

K.K. Rajan is with the Fast Reactor Technology Group \& and Engineering Services Group of IGCAR (telephone: +91 44274800 83, email:kkrajan@igcar.gov.in).

F. Michel is with the Commisariat a l'Energie Atomique et aux Energies Alternatives (CEA), Cadarache center DEN/DTN/STCP/LIET Bâtiment 202, 13108 St Paul-lez-Durance, France (telephone: +33 (0)4 422547 84, e-mail: michel.frederic@cea.fr). but here we focus on passive ALD. Some basic system requirements were proposed in 1990 by an IAEA specialists' meeting on steam generators [2] and here we restate the ones that have an impact on the proposed method:

- The sensitivity should be of the order of a few $\mathrm{g} / \mathrm{s}$.

- The detection time should be on the order of a few seconds.

- The false alarm rate should be less than one in two years.

A sensitivity of $1 \mathrm{~g} / \mathrm{s}$ for detection of a water-into-sodium leak was estimated by the IAEA, based on benchmark tests, to correspond to a signal to noise ratio (SNR) of $-17 \mathrm{~dB}$ in the noisiest steam generators studied at the time [3]. The SNR is defined as

$$
S N R=10 \log _{10}\left(\frac{P_{S}}{P_{b g}}\right)
$$

where $P_{S} / P_{b g}$ is the ratio of signal power to be detected to that of the background noise.

For a detection system which an output frequency of $1 \mathrm{~Hz}$ (in line with the second requirement), the specified false alarm rate corresponds to about $1.6 \times 10^{-8}$. By assuming Gaussian detector output and a $2 / 3$ voting logic between independent channels, this would correspond to a false alarm rate of $1.3 \times 10^{-4}$ for each channel.

Most of the works from the literature on passive ALD for SFRs uses methods where a substantial amount of knowledge on the leak noise to be detected is assumed. This knowledge has been used e.g. to create spectra, autoregressive models or wavelet transforms of the studied signals. The classification into background/injection have then been made using e.g. spectral distance measures, statistical measures, neural networks or support vector machines. See for example [3] and [4] for nice overviews of both classic and recent work on the topic.

However, given the complexity of acoustic signals it is unlikely that detailed leak noise knowledge can be easily credited when designing a system for a new plant. Even the normal background noise of a new plant will not be known beforehand and will be depending both on the operating point of the reactor and other loud systems running nearby. This implies that the detection system will need to be able to recognize several different noise types. To the requirements cited above, we therefore propose the addition of two desirable system properties:

- One part of the detector should be independent of reactor operating point.

- The detector should be able to recognize intermittent noises of normal operation that are not leaks. 
The motivation of both these properties is to reduce the amount of false alarms due to expected events such as change of reactor power or operation of components nearby, acoustically coupled to the steam generator, such as valves and pumps.

With the aim of investigating new methods capable of fulfilling all the above mentioned requirements, we here introduce a technique which is new to the field but has been both popular and successful within automatic speech recognition, namely the combination of hidden Markov models (HMMs) and Gaussian mixture models (GMMs).

This article is structured as follows: Section II contains a brief description of the basics of HMMs, GMMs and how they can be used. Then we go on to describing discriminating features based on the PSD in section III.A and introducing our new feature calculation scheme in section III.B. Section IV describes the IGCAR SOWART experiment and the obtained acoustic signals. Parametric studies on the proposed method based on these signals are described in section $\mathrm{V}$ and results on the detection performance of the method are shown in section VI. Discussion of the results is provided in section VII while conclusions and suggestions for future work are given in section VIII.

\section{HIDDEN MARKOV MODELS}

Hidden Markov models are statistical signal models based on the Markov property, i.e. that the probability of passing from one state to another is only dependent on the present state and not on the preceding history of states. Here, we follow along the lines of [5], to explain the basic properties of HMMs. Formally, if states at discrete points in time are labeled $s_{n}$, then a sequence of states is a Markov sequence if

$$
P\left(s_{n+1} \mid s_{n}, s_{n-1}, \ldots, s_{0}\right)=P\left(s_{n+1} \mid s_{n}\right)
$$

The probabilities for passing from one state to another are gathered in a transition matrix A with components $a_{i j}>0$ corresponding to the probability of passing from state $i$ to state $j$ in one time step. For a total of $N$ possible states, it is by definition required that

$$
\sum_{j=1}^{N} a_{i j}=1
$$

For a full description of the Markov sequence, an initial state vector, $\pi$ also needs to be specified, representing the probabilities of starting the sequence in each of the $N$ states.

The creation of a signal model from a Markov process is made by associating the state sequence with the signal output in some way. A hidden Markov model assumes that the states are not directly observable, but that each state has its own output probability distribution of so-called features that can be observed. In audio signal modeling, Gaussian mixture models are commonly used for this purpose. The distribution of observable features in each state is then given by a linear combination of $M$ Gaussian distributions, i.e.

$$
\boldsymbol{B}(\boldsymbol{x})=\sum_{m=1}^{M} b_{m} \frac{1}{(2 \pi)^{K / 2} \sqrt{\operatorname{det}\left(C_{m}\right)}} e^{-\frac{1}{2}\left(\boldsymbol{x}-\boldsymbol{\mu}_{m}\right)^{T} C_{m}^{-1}\left(\boldsymbol{x}-\boldsymbol{\mu}_{\boldsymbol{m}}\right)}
$$

where $b_{m}$ are weight factors, $K$ is the number of feature vectors in $\boldsymbol{X}, C_{m}$ is the covariance matrix of the features and $\mu_{m}$ are the Gaussian mean values [6]. The parameters of the complete model $\lambda(\mathrm{A}, \boldsymbol{B}, \boldsymbol{\pi})$ can be estimated by using a training algorithm, such as the Baum-Welch algorithm, on observed data [5].

Given a model $\lambda$ and a sequence of observations $\boldsymbol{x}$, it is possible to calculate exactly the conditional probability $P(x \mid \lambda)$, i.e. the probability that the observed output is generated by the model $\lambda$. This probability can, by Bayes rule, be written

$$
P(\boldsymbol{x} \mid \lambda)=P\left(x_{1} \mid \lambda\right) P\left(x_{2} \mid x_{1}, \lambda\right) \ldots\left(x_{t} \mid x_{t-1} \ldots x_{1}, \lambda\right)
$$

which can be calculated by a recursive algorithm [4]. Since all the factors are $<1$, this probability will become very small, even for relatively short feature sequences. For practical implementations, it is therefore advised to work with the logarithm of (5) [6].

\section{FEATURES FOR ACOUSTIC LEAK DETECTION}

A raw acoustic signal without any obvious piecewise structure is not suitable for direct modeling by the HMM+GMM method. To use this approach, the raw signal will instead have to be transformed in a suitable way, creating features that are characteristic of the monitored sounds. In the following sections, we will introduce a new feature calculation method which allows this to be done on industrial noise signals that, a priori, do not present the same obvious piecewise structure as do e.g. speech signals.

\section{A. Basic Features Based on Power Spectral Density}

The Power Spectral Density (PSD) of a signal $x(t)$ is a function in frequency space representing the power transmitted within each frequency band of the signal. An estimate of the PSD during a time $T$ is given by

$$
X(f, T)=\frac{1}{T}\left|\int_{-T / 2}^{T / 2} x(t) e^{-i 2 \pi f t} d t\right|^{2}
$$

By performing successive estimates with a sliding time window it is possible to monitor the spectral evolution of the signal. Each time window may also be subdivided into overlapping sub windows in order to decrease the spectral uncertainty by averaging over several estimates of the PSD. This approach is known as the Welch method [7] and will be used here.

Furthermore, to avoid that the detector reacts on pure signal power changes (which can be expected for reactor operating point changes), we propose a normalization such that

$$
\sum_{i} X\left(f_{i}, T_{n}\right)=1
$$

It was suggested in [8] that a sum of the PSD at selected frequencies would be a suitable feature for acoustic leak detection. The frequencies would be selected, before starting the detection system, as those presenting the largest contrast between a known leak/injection signal and a known background signal. This type of leak discriminating feature was called PSDSUM, i.e.

$$
\operatorname{PSDSUM}(n)=\sum_{i \in I_{\max }} X_{n}\left(f_{i}, T_{n}\right)
$$

where $n$ is enumerating the successive time windows of length $T_{n}$ and $I_{\max }$ is a set of local maxima of

$$
X_{\text {inj }}\left(f_{i}, T_{\text {inj }}\right)-X_{b g}\left(f_{i}, T_{b g}\right)
$$


where $X_{i n j}$ and $X_{b g}$ represent the reference spectra of known injection and background noises.

In order to detect signal changes by a PSDSUM approach without a priori knowledge on the noise to be detected, it was proposed in [9] to monitor the difference

$$
X R\left(f_{i}, n\right)=X_{n}\left(f_{i}, T_{n}\right)-X_{\text {ref }}\left(f_{i}, T_{\text {ref }}\right)
$$

where $X_{n}\left(f_{j} T_{n}\right)$ is the PSD estimated in the current time window $T_{n}$ of the monitored signal and $X_{\text {ref }}\left(f_{j}, T_{r e f}\right)$ is a reference PSD. Frequency components are selected online as those currently presenting the largest difference to their reference background value. The generalization of (8) then becomes

$$
\operatorname{XRSUM}(n)=\sum_{j \in J_{\max }(n)} X R\left(f_{j}, n\right)
$$

where the set of local maximum deviation frequencies $J_{\max }(n)$ may change over time. Note that this feature is sensitive to all deviations from the reference.

\section{B. A New Feature for the HMM+GMM Method}

By combining measures on known characteristic frequencies and the currently most deviating frequencies, we propose a new feature that is piecewise in nature and thus suitable for modeling by HMMs. The proposed feature can be written

$$
F_{k}(n)=X R\left(f_{i_{k}}, n\right)+a *\left(i_{k}-j_{k}(n)\right)
$$

where $X R$ is created from PSDs normalized according to (7), $i_{k} \in I_{\max }$ is a set of local maxima ordered by power in a reference PSD and $j_{k} \in J_{\max }$ is an set of local maxima in the current deviation from the reference PSD, also ordered by power. The number $k$ enumerates the local maxima, with $k=$ 1 representing the largest maximum and so on. The largest $k$ value is equal to the $K$ of (4). The number $a$ is chosen so that a shift of one frequency band yields a total feature shift which is much larger than the expected variation in power density, resulting in a piecewise structure of the feature. A typical evolution of the proposed feature, demonstrating a signal change is shown in figure 1.

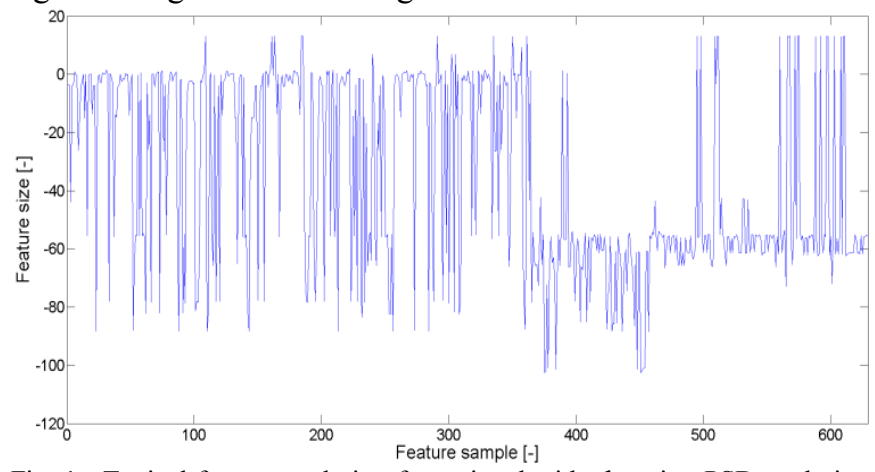

Fig. 1. Typical feature evolution for a signal with changing PSD evolution structure after about 360 samples.

A histogram of the feature will then resemble the PSD as shown in figure 2, yielding a link (although not obvious) between a noise type and its hidden Markov model.
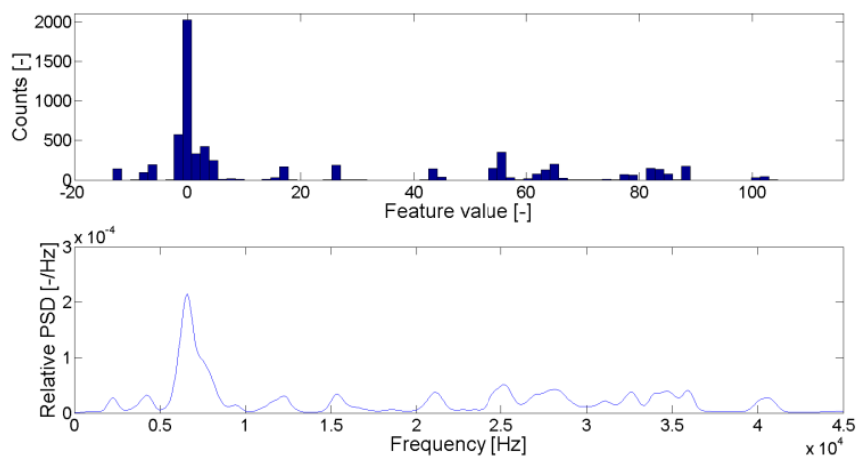

Fig. 2. Histogram of a noise model feature (upper panel) and the PSD of the same noise signal (lower panel).

By training a $\mathrm{HMM}+\mathrm{GMM}$ on the proposed feature sequence of a known signal and then reapplying the trained model to a test signal by successively calculating the logarithm of (5), a detector is obtained. The decision time of this detector can be changed by varying the length of the feature sequence used in (5).

The implementation of the feature calculation scheme and the HMM+GMM method was made in MATLAB by use of a pattern recognition library developed at KTH [6].

\section{THE SOWART EXPERIMENT (IGCAR)}

In collaboration between the IGCAR and the CEA on the safety of SFR steam generators, a number of sodium-water reaction experiments were carried out in the Sodium Water Reaction Test rig (SOWART) facility of IGCAR [10]. In these experiments, steam/water was injected into sodium through a calibrated injection nozzle, directly impinging on a $9 \mathrm{Cr}-1 \mathrm{Mo}$ steam generator tube. The main purpose of these experiments was to study the effect of wastage on the tube exposed to the water/steam jet under typical operating conditions of a SFR system [10].

The acoustic noise generated was measured using three accelerometer instrumented wave-guides welded to the test section. The obtained recordings may be useful in the characterization of the sodium-water reaction acoustic signature and the development of ALD systems.

In total 10 injections were made according to the operating conditions given in Table I. The estimated flow rates corresponding to all injection conditions were less than $1 \mathrm{~g} / \mathrm{s}$.

TABLE I. INJECTION EXPERIMENT OPERATING CONDITIONS

\begin{tabular}{llll} 
Test ID & $\begin{array}{l}\text { Sodium and } \\
\text { steam } \\
\text { temperature }\left[{ }^{\circ} \mathbf{C}\right]\end{array}$ & $\begin{array}{l}\text { Steam } \\
\text { pressure } \\
{[\mathbf{b a r}]}\end{array}$ & $\begin{array}{l}\text { Injection } \\
\text { hole diameter } \\
{[\mathbf{m m}]}\end{array}$ \\
\hline SW1 & 340 & 172 & 0,1 \\
SW2 & 500 & 172 & 0,2 \\
SW3 & 350 & 172 & 0,1 \\
SW4 & 420 & 172 & 0,2 \\
SW5 & 420 & 172 & 0,2 \\
SW6 & 480 & 172 & 0,2 \\
SW7 & 480 & 172 & 0,1 \\
SW8 & 480 & 172 & 0,2 \\
SW9 & 490 & 172 & 0,2 \\
SW10 & 500 & 172 & 0,2 \\
\hline
\end{tabular}


The recordings used in the present study were those of SW1, SW2, SW3, SW4 and SW6. With three channels per test, this yielded in total 15 acoustic signals. As the signals recorded from tests SW9 and SW10 contained too short regions of pure background noise before injection, they were not suitable for the present study. Furthermore, the signals of SW5, SW7 and SW8 were similar to those of SW3 and SW4 and therefore discarded in this study.

\section{PARAMETRIC STUdies}

The following signal processing parameters were used: The sampling rate of the SOWART acoustic signals was 200 $\mathrm{kHz}$. Sliding time windows of length $T=10 \mathrm{~ms}$ were used and the Welch method was set up with sub windows of $4 \mathrm{~ms}$ overlapping each other by $2 \mathrm{~ms}$. Three local maxima were used in (12), i.e. $k$ ranged from 1 to 3 .

Each signal was divided into three parts, were the first part served as training data for the background noise model, the middle part (containing the transition between pure background noise and injection noise) served as testing data and the last part served as training data for the injection noise model. The models were trained by the Baum-Welch method to create ergodic, infinite-duration Markov chains with Gaussian mixture output distributions. The training data were segmented by chunks of 100 time windows and the number $a$ of (12) was set to 0.3 since this choice was found to provide satisfactory results for a first study.

The remaining free parameters of the presented method were then the number of GMM components and the number of HMM states. The influence of these numbers on the resulting model was studied explicitly by applying trained models on the test signals. In figures 3-4, the resulting log probabilities for SW2 are shown as function of the number of GMM components and HMM states.

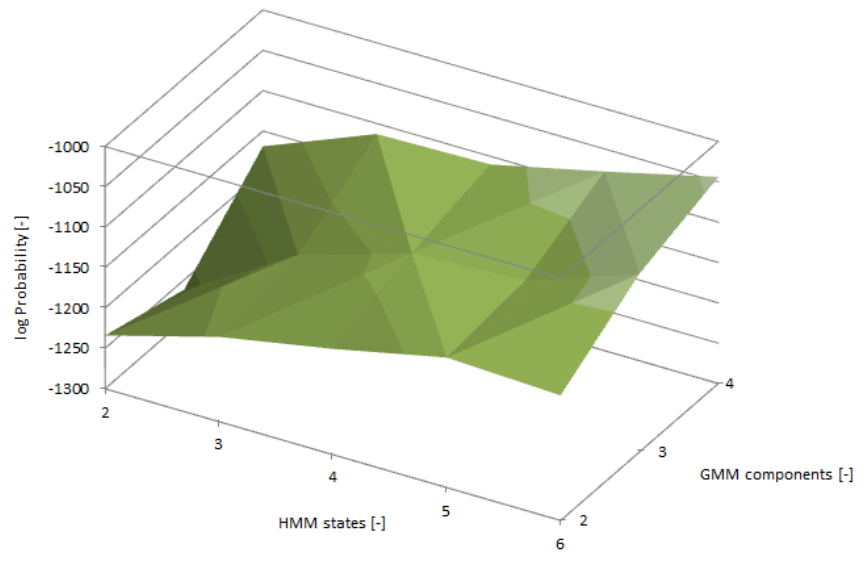

Fig. 3. SW2, study of background model log probability as function of the number of GMM components and the number of HMM states

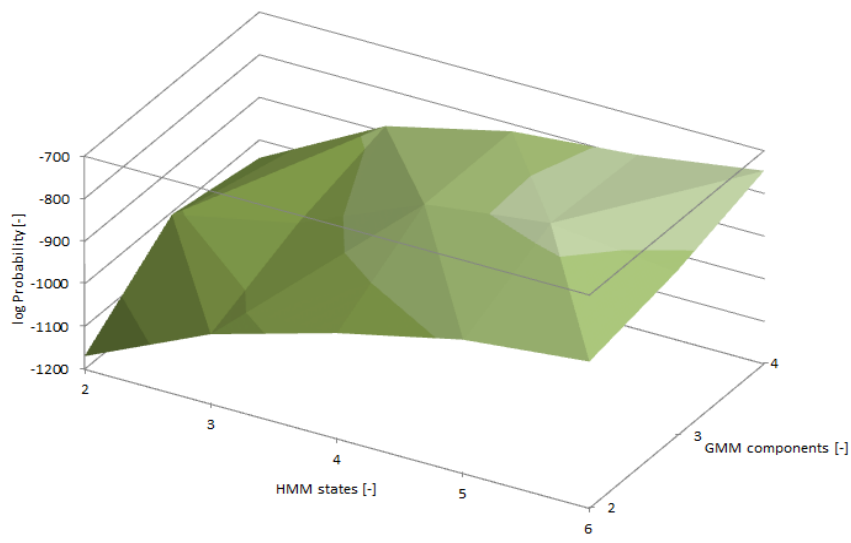

Fig. 4. SW2, study of injection model log probability as function of the number of GMM components and the number of HMM states

The tendency of increased model probability with both the number of GMM components and HMM states was present in all studied signals. However, training problems due to lack of data also appeared, notably with increasing number of GMM components. We therefore chose to work with 3 GMM components and $6 \mathrm{HMM}$ states. For this model size, training problems still occurred occasionally but to an extent that was judged acceptable for this work.

\section{RESULTS}

\section{A. Detection Capability in SOWART Data}

In figures 6-11, the testing parts of recordings SW1, SW2 and SW6 with corresponding model log probabilities are shown. The detector decision time was set to 1 second. All detector outputs are in the figures normalized to have zero mean and variance one during the first 60 seconds of the signal. In one case, an artificial signal power change before injection was introduced, to demonstrate the detector invariance to such changes.

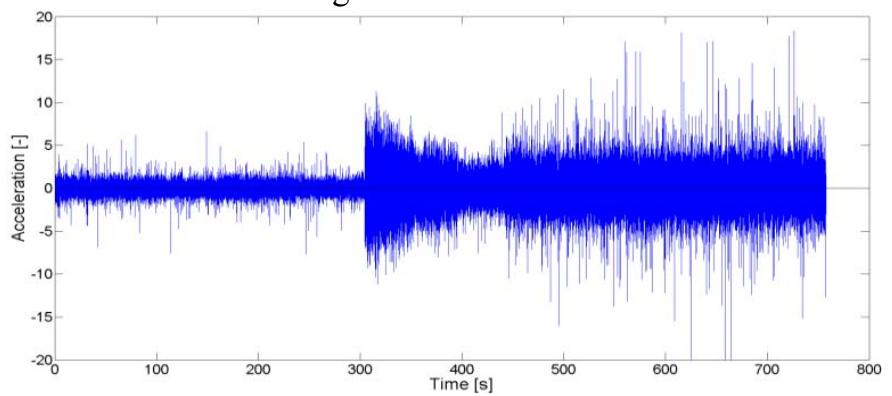

Fig. 6. SW1, raw signal at accelerometer 1 .

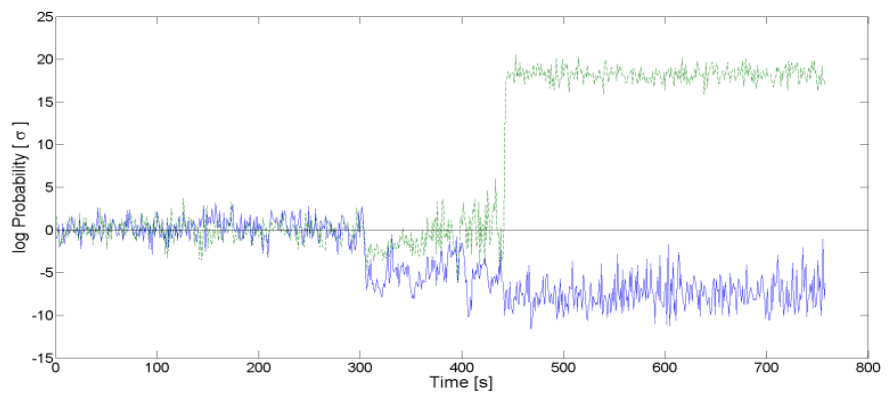

Fig. 7. SW1, log probabilities of background model (solid line) and injection model (dashed) of the accelerometer 1 signal. 


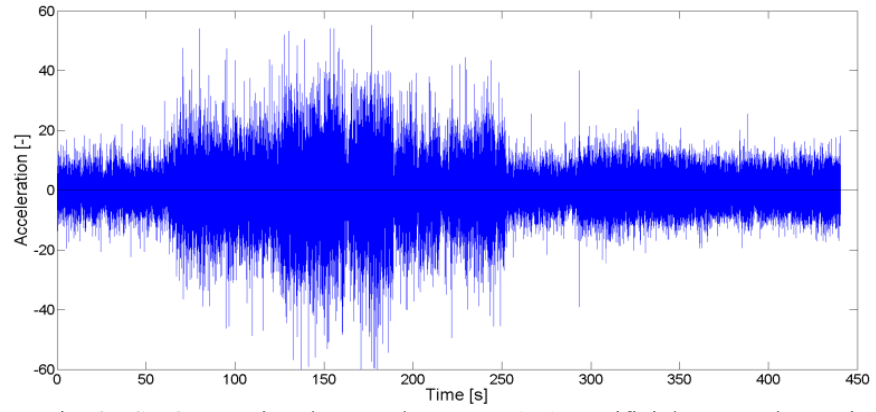

Fig. 8. SW2, raw signal at accelerometer 1 . An artificial power change is introduced before injection.

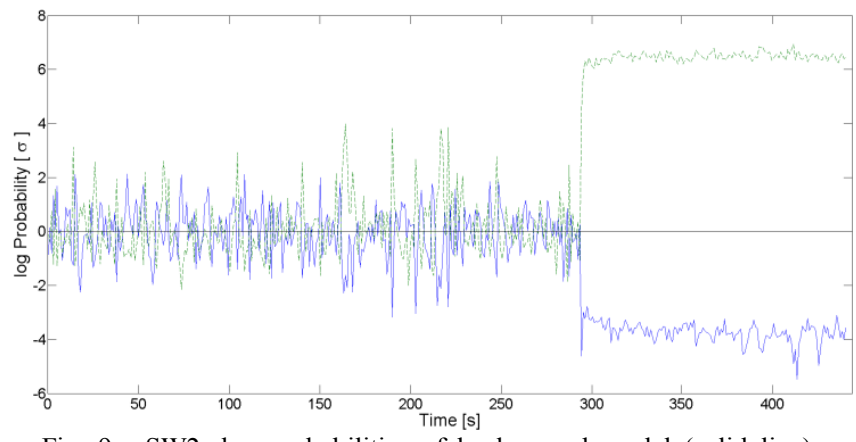

Fig. 9. SW2, log probabilities of background model (solid line) and injection model (dashed) of the accelerometer 1 signal.

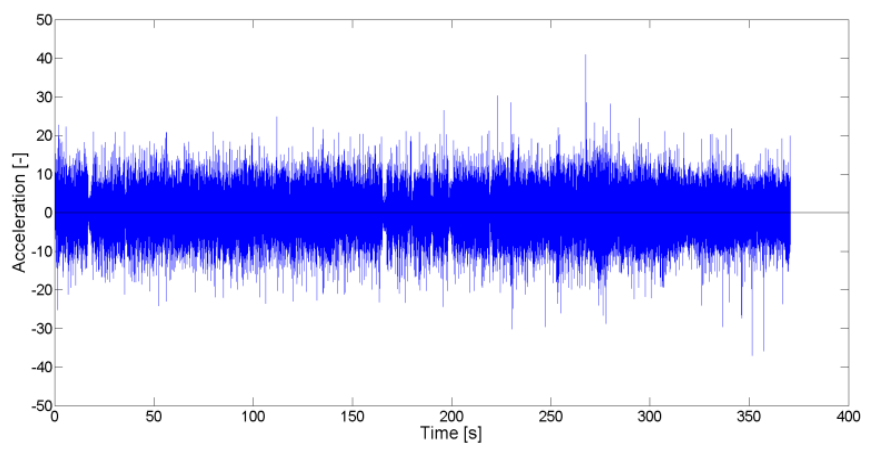

Fig. 10. SW6, raw signal at accelerometer 1.

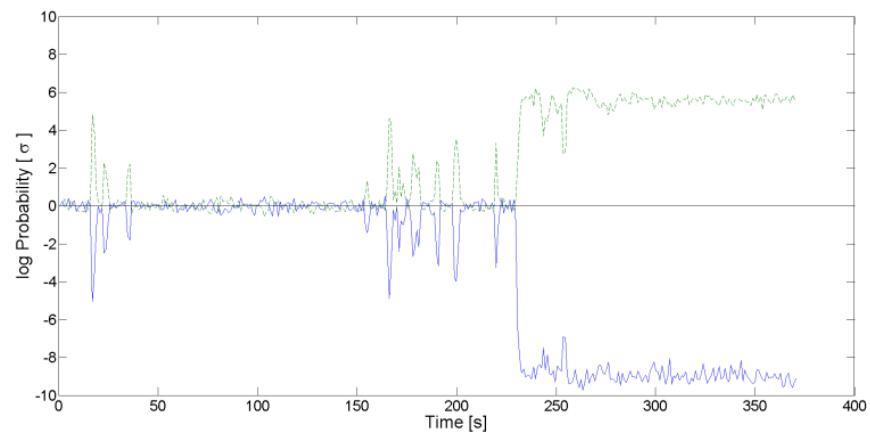

Fig. 11. SW6, log probabilities of background model (solid line) and injection model (dashed).

It is clear that both the background and injection noise models are fully capable of detecting the injections, while being signal power invariant.

Two types of anomalies can be identified in the detector output; the first type is seen for the injection model in SW1 which seems to initially change in the wrong direction. In SW6, a series of spikes appear before injection, seemingly correlated with dips in signal energy seen in the raw signal. The first anomaly type will be further discussed in section VII while the second type is assumed to be something which can occur also in a real system.

\section{B. False Alarm Rate}

The false alarm rate of a real passive acoustic detection system based on training of background noise models is dependent both on the statistical properties of the detector output and the long-term characteristics of the background noise. E.g. if the plant over time generates a lot of unrecognized noises, the false alarm rate will be higher until these noises have been learnt by the system. To estimate the magnitude of this problem for a new reactor system is more or less impossible. For the present study, we can however study the detector output during the pure background noise regions and estimate the false alarm rate for the SOWART facility. By concatenating the HMM background model detector output before injection in all test signals and making a histogram of this vector, the resulting distribution can be analyzed. The resulting raw data vector and its histogram are shown in figure 12. Some different background noise modes are visible, but it seems reasonable to make the assumption that the detector output is approximately Gaussian. This implies that it will be possible to control the false alarm rate in a straightforward way. By allowing for a $1.5 \sigma$ drift, the false alarm rate goal of $1.3 \times 10^{-4}$ identified in section I then corresponds to a threshold at about $4.5 \sigma$ from the mean detector output level.
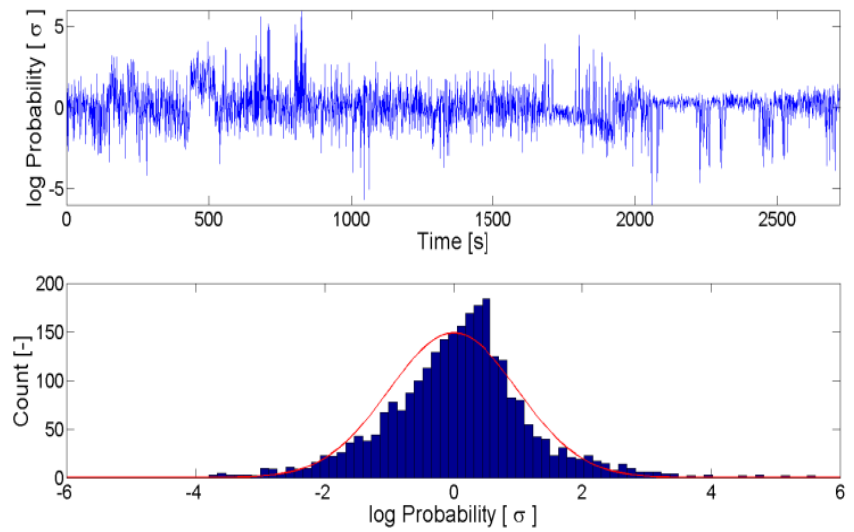

Fig. 12. Raw data and histogram of HMM background model detector output in background region of all testing signals. A standard normal distribution with equal area is added to the histogram for comparison.

\section{Detection Capability at Low SNR}

We now turn to investigation of the detection performance as function of SNR. Primarily, we study the background model detector, i.e. the solid line in figures 7, 9 and 11. As a first measure of detector performance we take the area under the receiver operating characteristic (ROC). Since the false positive rate for a passive ALD system needs to be very low, this area should be close to unity. We examined this measure as function of the SNR for each test signal by identifying one large region of pure background noise and one region of background + injection noise and simply counting the positive alarm rates in each region. 
As acoustic absorption was seen on injection onset in several signals, probably due to the appearance of hydrogen bubbles in the sodium, the SNR was estimated from the PSDs according to:

$$
S N R=10 \log _{10}\left(\frac{P_{i n j+b g}-\left(P_{b g}-P_{a b s}\right)}{P_{b g}-P_{a b s}}\right)
$$

where $P_{i n j+b g}$ is the signal power in the injection region, $P_{b g}$ is the signal power in the background region, and $P_{a b s}$ is the absorbed acoustic power during injection. The absorbed power was crudely estimated as the total power density decrease in the frequencies where the injection region PSD was smaller in magnitude than the background region PSD.

The area under ROC as function of SNR for the background noise deviation detector with a decision time of one second is shown in figure 13 .

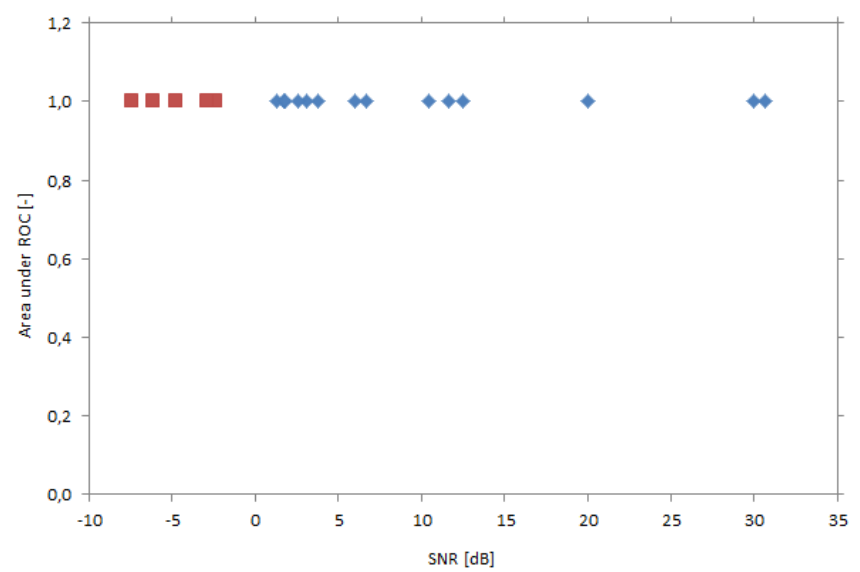

Fig. 13. Area under ROC curve as function of SNR for the background model detector with $1 \mathrm{~s}$ decision time. The maximal area under a ROC curve is normalized to be 1 . The data points at negative SNR (squares) were created by adding the background noise vector with arbitrary phase shift to the training and testing signals.

From figure 13 it is clear that the detector quality is almost acceptable for all studied recordings, but it is not possible to deduce any trend towards lower SNRs. We now define a detection margin respecting the false alarm rate goal, in analogy with the one of [3], as:

$$
D M_{4.5 \sigma}=20 \log _{10}\left(\frac{d_{S}}{d_{b g}+4.5 \sigma}\right)
$$

where $d_{s}$ and $d_{b g}$ are the average detector outputs in known injection and background regions of the signal. This detection margin is shown as function of SNR in figure 14.

Three outlier points at significantly higher detection margin were omitted from the figure. These points represented cases where training problems occurred, resulting in a large or even positive log probability for the background noise. Furthermore, six additional data points at negative SNR were created artificially. This was achieved by adding a circular repetition of the background noise vector with arbitrary phase shift to the training and testing signals of the six raw data points at lowest SNR.

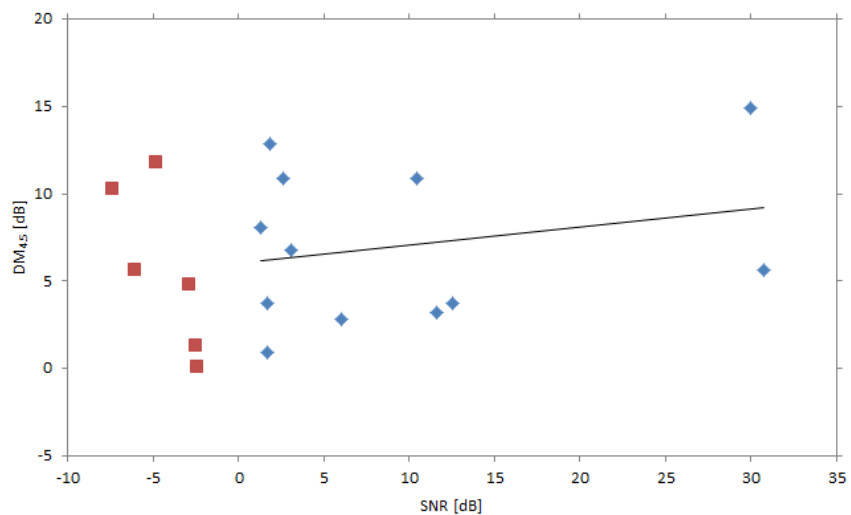

Fig. 14. Detection margin to $4.5 \sigma$ versus SNR for the background model detector with $1 \mathrm{~s}$ decision time. The data points at negative SNR (squares) were created by adding the background noise vector with arbitrary phase shift to the training and testing signals. Three data points with detection margin above $20 \mathrm{~dB}$ are omitted and a least squares fit of a linear function to the raw data points (diamonds) is included.

The detection margin dependency on SNR found from figure 13 is relatively small. However, since the spread in is large, it is reasonable to assume that the practical limit of detection occurs already at a few $\mathrm{dB}$ below zero. By increasing the decision time of the detector from $1 \mathrm{~s}$ to $5 \mathrm{~s}$, i.e. using longer sequences in the calculation of (5), the margin can be increased, as shown in figure 15. Here however, an outlier point at negative margin appears. This seems to be due to background noise variations observed before injection in one of the SW1 signals, yielding a high value of $\sigma$.

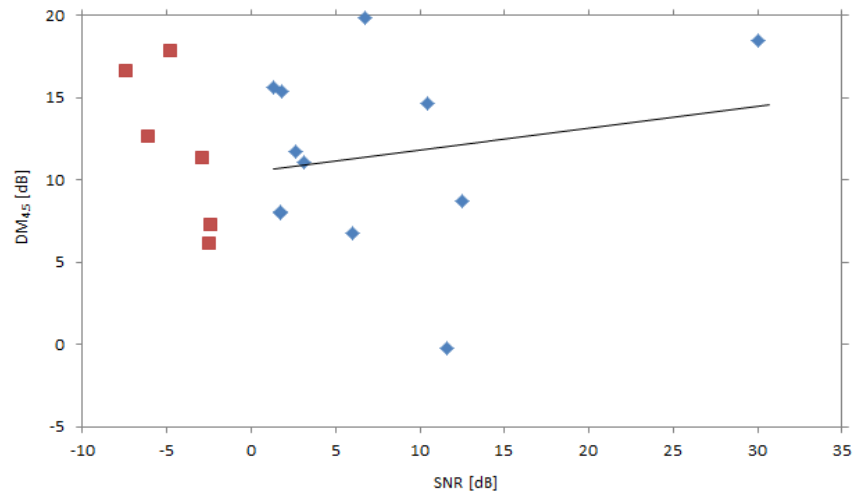

Fig. 15. Detection margin to $4.5 \sigma$ versus SNR for the background model detector with $5 \mathrm{~s}$ decision time. The data points at negative SNR (squares) were created by adding the background noise vector with arbitrary phase shift to the training and testing signals. Three data points with detection margin above $20 \mathrm{~dB}$ are omitted and a least squares fit of a linear function to the raw data points (diamonds) is included.

As a final demonstration of the HMM detector capability, we use knowledge of the injection noise and create a new detection measure as the difference between the injection and background model log probabilities. The results for this detector are shown in figure 16. Also here, background noise variations before injection in one of the SW1 signals yielded a high value of $\sigma$ and consequently a point at negative detection margin. 


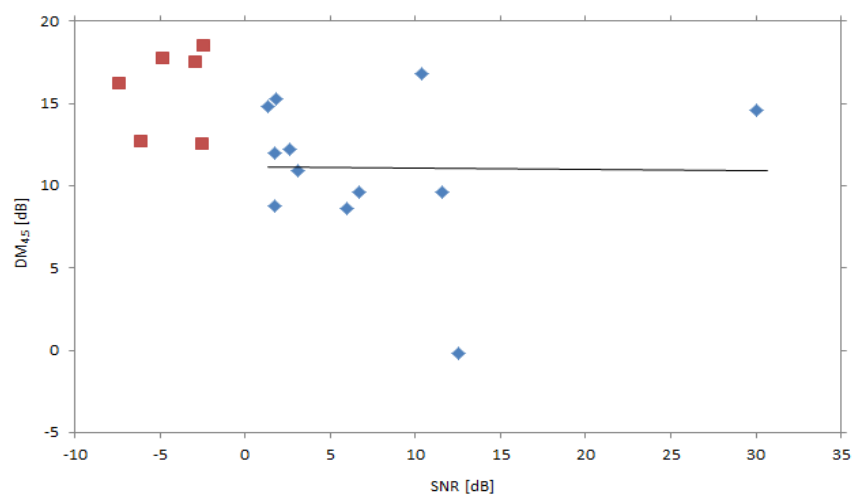

Fig. 16. Detection margin to $4.5 \sigma$ versus SNR for the injectionbackground difference detector with $5 \mathrm{~s}$ decision time. The data points at negative SNR (squares) were created by adding the background noise vector with arbitrary phase shift to the training and testing signals. Three data points with detection margin above $20 \mathrm{~dB}$ are omitted and a least squares fit of a linear function to the raw data points (diamonds) is included.

\section{DISCUSSION}

In a relatively small mechanical system designed for injection experiments, it is difficult to exclude the possibility that the injections are detected through vibrations propagated directly from the injection mechanism itself rather than through jet noise and sodium-water reaction noise from the leak as would be the case in a real system.

The interpretation of the proposed feature calculation method is not obvious as it includes addition of frequency and power spectral density deviation, with the number $a$ as scaling constant. It obviously generates a feature that is suitable for $\mathrm{HMM}+\mathrm{GMM}$ combination, but development of schemes with a clearer physical interpretation are of interest.

Furthermore, when a signal is changing, it is not certain that the conditional probability of (5) will change as we expect. Basically, we expect the background noise probability to go down and the injection noise probability to go up at injection onset. An interesting counterexample is seen in figure 7 , where the injection seems to start in one flow regime and then change to another regime, more similar to that of the training data. The result is that initially, both the background noise and the injection noise probabilities go down.

The uncertain magnitude of the model probability changes is seen in figures 14, 15 and 16 as the detection margins have a relatively large spread and that outliers occur for models with high variance. Also, the SNR estimation of (13) is problematic as an unknown amount of acoustic absorption seems to occur during injections. This raises a question of the confidence level of the detector. On the other hand; the simple output distribution of the detector signal shown in figure 12 and the weak dependency on SNR observed in figures 14-16 are still properties that are encouraging for future work on this type of detector.

Earlier results, primarily from [3] have often focused on the low SNR capability and have also reached impressive results such as detection down to less than $-20 \mathrm{~dB}$. For the proposed method it seems probable that a limit of around -17 $\mathrm{dB}$ could be reached with acceptable false alarm rate, although with a decision time that might be somewhat too long. However, the fact that very few earlier works report estimates on SNR limit, detection time and false alarm rates together makes thorough method comparison difficult. Differences between best and worst case performance are also seldom mentioned. One important explanation for this lack of estimates is that real plant data are so few and that background and leak noises of a new plant are extremely difficult to predict.

\section{CONCLUSIONS}

By comparing the proposed method with the requirements listed in section I of this paper the following conclusions can be drawn:

Injection rates below $1 \mathrm{~g} / \mathrm{s}$ are clearly detected in all recordings and the false alarm criterion can be met. However, the SNRs of the SOWART acoustic signals are higher than expected in a real system. Crude extrapolation of the results to lower SNR suggests that the false alarm rate criterion can be met also in a more realistic sound environment by increasing the detector decision time and/or crediting injection noise models. However, the confidence level of the method is an open question, as it is for other methods presented in the literature.

Given that the detector output is approximately Gaussian, that the method will be able to model several noise types, that signal energy changes can successfully be discarded and that the proposed feature calculation scheme yields promising results, also without a priori knowledge on the injection noise, the method seems worthy of further research and development. Such R\&D should include improvement of the feature calculation scheme, validation of the method at low SNR and further investigation of the relation between the feature calculation scheme, model size and performance of the detector.

\section{ACKNOWLEDGMENTS}

The authors express their sincere gratitude to the Director of IGCAR for his encouragement throughout the CEAIGCAR collaborative program and to the staff members of the Fast Reactor Technology Group of IGCAR for their support in carrying out the SOWART experiments.

We also wish to thank Pierre-Augustin Grivelet of RMS Signal \& Innovation for interesting and valuable discussions on the ALD problem.

\section{REFERENCES}

[1] Generation IV International Forum, "Generation IV Goals", https://www.gen-4.org/gif/jcms/c_40472/technology-goals, Date of latest access: 2015-03-18

[2] J. Voss, P.J. Thomas and J.P. Girard, "Review of the Common European R \& D Programme on Acoustic Leak Detection for Steam Generators", IAEA Specialists' Meeting on Steam Generators: Acoustic Detection of In-Sodium Water Leaks, Aix-en-Provence, 1 - 3 October, 1990

[3] International Atomic Energy Agency, "Acoustic signal processing for the detection of sodium boiling or sodium-water reaction in LMFRs", IAEA-TECDOC-946, 1997 
[4] N. Matta, Y. Vandenboomgaerde and J. Arlat, "Supervison and Safety of Complex Systems", John Wiley \& Sons, Inc., 2012, ISBN: 978-184821-413-2

[5] L. R. Rabiner, "A tutorial on hidden Markov models and selected applications in speech recognition", Proceedings of the IEEE, Vol. 77, No. 2, pp. 257-285, 1989

[6] A. Leijon, "Pattern recognition - fundamental theory and exercise problems", KTH Electrical Engineering, 2010

[7] P.D Welch, "The use of fast fourier transform for the estimation of power spectra: A method based on time averaging over short modified periodograms", IEEE Transactions on Audio Electroacoustics, Vol. AU-15, pp.70-73, 1967

[8] G.S. Srinivasan, O.P. Singh and R. Prabhakar, "Leak noise detection and characterisation using statistical features", Annals of Nuclear Energy, Vol. 27, pp. 329-343, 2000

[9] A. Riber Marklund and F. Michel, "Application of a new passive acoustic leak detection approach to recordings from the Dounreay prototype fast reactor", submitted for publication

[10] S. Kishore, A. Ashok Kumar, S. Chandramouli, B.K. Nashine, K.K. Rajan, P. Kalyanasundaram, S.C. Chetal, "An experimental study on impingement wastage of Mod 9 $\mathrm{Cr} 1$ Mo steel due to sodium water reaction", Nuclear Engineering and Design, Vol. 243, pp. 49-55, 2012 